\title{
Birefringence measurement in the terahertz range based on double Fourier analysis
}

\author{
Federico Sanjuan, ${ }^{1,2, *}$ Alexander Bockelt, ${ }^{1}$ and Borja Vidal ${ }^{1}$ \\ ${ }^{1}$ Nanophotonics Technology Center, Universitat Politècnica de València, Camino de Vera, s.n., Valencia 46022, Spain \\ ${ }^{2}$ CIOp (Conicet-CIC), cno. Parque Centenario e/505 y 508, Buenos Aires 1897, Argentina \\ *Corresponding author: fedesanjuan@yahoo.com.ar
}

Received December 2, 2013; revised December 31, 2013; accepted January 4, 2014;

posted January 6, 2014 (Doc. ID 202417); published February 6, 2014

\begin{abstract}
A method for obtaining the average refractive indexes of a birefringent material in the terahertz region in a single measurement with a standard terahertz time-domain spectrometer is presented. The method is based on processing the frequency-domain interference between terahertz pulses and echoes through the Fourier transform of the terahertz spectrum. The technique also allows the determination of the optical axis orientation of the material by making two measurements with different angles of the sample optical axis. (c) 2014 Optical Society of America

OCIS codes: (160.4760) Optical properties; (260.1440) Birefringence; (140.3490) Lasers, distributed-feedback; (300.6495) Spectroscopy, terahertz.

http://dx.doi.org/10.1364/OL.39.000809
\end{abstract}

Birefringence measurements can be used to derive a wide range of parameters, such as mechanical stress in plastic components, molecular chain alignment in liquid crystals, and orientation of fibers in paper, composites, wood, and other materials [1-3]. Different techniques have been applied to characterize birefringent materials, such as visible and near-infrared (NIR) light, x-ray diffraction (XRD), and terahertz radiation. Terahertz spectroscopy allows the characterization of many materials of industrial interest, such as plastics, wood, textiles, and paper that are opaque to visible and NIR light, providing large penetration depth. Besides, terahertz technology offers a safer approach than XRD since it is nonionizing [1].

The birefringence of a material and the orientation of the optical axis in the terahertz band have been obtained by different techniques. The conventional procedure is based on a standard terahertz time-domain spectroscopy (TDS) setup performing at least a reference measure plus three others with three different angles of the sample for achieving both refractive indices and optical axis orientation $[4,5]$. However, it requires at least four measurements, which can be both slow and cumbersome. A faster way to obtain average refractive indices is by measuring the delay between the peaks of the main pulses and their respective echoes in the temporal domain [6]. This method can only be applied when the delays between pulses and echoes are long enough to distinguish them. When the delay is in the order of the time pulse width, which is generally around $1 \mathrm{ps}$, a direct time analysis is not possible. Additionally, the determination of the orientation of the optical axis as a relation of the temporal pulse amplitudes [1] is not possible when diattenuation occurs. Another proposal to determine both refractive indices and optical axis in a single measure is developed in [7], where the terahertz spectrometer employs circularly polarized terahertz waves and a polarization-sensitive detector that measures both components of the electric field simultaneously. However, this method is not applicable with a standard terahertz spectrometer.

In this Letter, we propose a technique for measuring the average refractive indices of birefringent materials in a single measurement and a method for obtaining the optical axis orientation in materials with constant refractive index in the terahertz range, such as various plastics, paper, or wood [3,6]. The method relies on the information obtained from the interference generated by the temporal pulses and echoes in the frequency domain. In particular, the proposed method is based on calculating the discrete Fourier transform (DFT) of the squared modulus of the detected frequency-domain electric field.

Figure 1 shows the general interaction of a linearly polarized electric field on a birefringent sample. The electric field is projected onto the ordinary and extraordinary axis and affected by a different delay and attenuation. In the detector, both components of the electric field are projected onto its polarized direction.

The electric field after a birefringent material in the frequency domain can be expressed as Eq. (1) []], where a continuous signal is considered in the analysis for the sake of simplicity:

$$
\begin{aligned}
& E_{T}(\omega) \\
& =\left|E_{i}(\omega)\right| \cos ^{2}(\theta) e^{j \varphi_{i}(\omega)} T_{o}(\omega) G_{o}(\omega, d) \sum_{m=0}^{+\infty}\left(r_{o}(\omega) G_{o}^{2}(\omega, d)\right)^{m} \\
& +\left|E_{i}(\omega)\right| \sin ^{2}(\theta) e^{j \varphi_{i}(\omega)} T_{e}(\omega) G_{e}(\omega, d) \sum_{m=0}^{+\infty}\left(r_{e}(\omega) G_{e}^{2}(\omega, d)\right)^{m},
\end{aligned}
$$

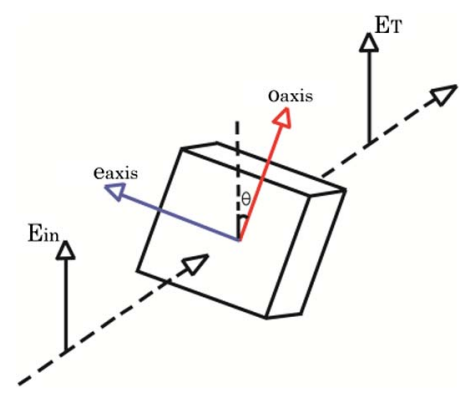

Fig. 1. Projection of a terahertz electric field onto a birefringent material. 
where $\left|E_{i}(\omega)\right| e^{j \varphi_{i}(\omega)}$ is the incident electric field; $T(\omega)=$ $\left(4 n(\omega) /(n(\omega)+1)^{2}\right)$ and $r(\omega)=\left((n(\omega)-1)^{2} /(n(\omega)+1)^{2}\right)$ are the square of the transmission and reflection coefficient, respectively; $G(\omega)=e^{-j n(\omega) \omega d / c}$ is the sample propagation coefficient; $\omega$ is the angular frequency; $\theta$ is the angle between the sample optic axis and the antenna axis; $d$ is the sample thickness; and $m$ is the number of echoes due to the Fabry-Perot effect. In Eq. (1) the first term is affected by the ordinary refractive index $\left(n_{o}\right)$ and the second by the extraordinary $\left(n_{e}\right)$.

Equation (2) is the squared modulus of Eq. (1), assuming the refractive indices are frequency independent and considering only one echo in each term, since further echoes will generally be weaker:

$$
\begin{aligned}
\left|E_{T}(\omega)\right|^{2}= & a_{1}\left(\theta, n_{e}, n_{o}\right)\left|E_{i}(\omega)\right|^{2} \cos \left(\left(n_{o}-n_{e}\right) \frac{\omega d}{c}\right) \\
& +a_{2}\left(\theta, n_{e}, n_{o}\right)\left|E_{i}(\omega)\right|^{2} \cos \left(\left(3 n_{o}-n_{e}\right) \frac{\omega d}{c}\right) \\
& +a_{3}\left(\theta, n_{e}, n_{o}\right)\left|E_{i}(\omega)\right|^{2} \cos \left(\left(n_{o}-3 n_{e}\right) \frac{\omega d}{c}\right) \\
& +a_{4}\left(\theta, n_{e}, n_{o}\right)\left|E_{i}(\omega)\right|^{2} \cos \left(2 n_{o} \frac{\omega d}{c}\right) \\
& +a_{5}\left(\theta, n_{e}, n_{o}\right)\left|E_{i}(\omega)\right|^{2} \cos \left(2 n_{e} \frac{\omega d}{c}\right) \\
& +a_{6}\left(\theta, n_{e}, n_{o}\right)\left|E_{i}(\omega)\right|^{2} \cos \left(3\left(n_{o}-n_{e}\right) \frac{\omega d}{c}\right) \\
& +a_{7}\left(\theta, n_{e}, n_{o}\right)\left|E_{i}(\omega)\right|^{2} .
\end{aligned}
$$

$\left|E_{i}(\omega)\right|^{2}$ is the free-air spectrum of the terahertz signal, which is assumed to be constant in frequency. Applying the Fourier transform (FT) to Eq. (2), Eq. (3) is obtained. We can see in Eq. (3) that besides the continuous component, six terms are present at the receiver. These are caused by every combination of the four pulses present in the time domain after the material. These six terms are the convolution between a delta in the origin with the FT of the interference (cosine):

$$
\begin{aligned}
Y_{T}(t)= & H_{1}\left(\theta, n_{e}, n_{o}\right) \delta\left(t-\left|n_{o}-n_{e}\right| \frac{d}{c}\right) \\
& +H_{2}\left(\theta, n_{e}, n_{o}\right) \delta\left(t-\left|3 n_{o}-n_{e}\right| \frac{d}{c}\right) \\
& +H_{3}\left(\theta, n_{e}, n_{o}\right) \delta\left(t-\left|n_{o}-3 n_{e}\right| \frac{d}{c}\right) \\
& +H_{4}\left(\theta, n_{e}, n_{o}\right) \delta\left(t-2 n_{o} \frac{d}{c}\right) \\
& +H_{5}\left(\theta, n_{e}, n_{o}\right) \delta\left(t-2 n_{e} \frac{d}{c}\right) \\
& +H_{6}\left(\theta, n_{e}, n_{o}\right) \delta\left(t-3\left|n_{o}-n_{e}\right| \frac{d}{c}\right) \\
& +H_{7}\left(\theta, n_{e}, n_{o}\right) \delta(0) .
\end{aligned}
$$

Figure 2 shows a numerical simulation of the absolute value of the DFT of Eq. (2) assuming a bandwidth up to $3 \mathrm{THz}$ with a frequency resolution of $5 \mathrm{GHz}$, $n_{e}=3.07, n_{o}=3.39, d=1 \mathrm{~mm}$, and $\theta=45^{\circ}$.

Due to the finite length of the signal, the peaks in Fig. 2 are not ideal deltas. The two peaks near the origin correspond to the first and six terms of Eq. (3). The more similar $n_{o}$ and $n_{e}$ are, the closer these peaks will be to the origin. The refractive indices can be derived from the other four pulses. In particular, the inner pulses correspond to the fourth and fifth terms of Eq. (3) that presents time shifts of $T_{1}=2 n_{o} d / c$ and $T_{2}=\overline{2} n_{e} d / c$. From these, both refractive indices can be obtained independently as $n_{o}=T_{1} c / 2 d$ and $n_{e}=T_{2} c / 2 d$.

In practice, when $n_{o}$ is close to $n_{e}$, the second and fourth terms of Eq. (3) are very close and also the third and fifth terms. Therefore, depending on the terahertz spectrometer resolution, it is possible that only two peaks can be discriminated instead of the four given by theory and shown in Fig. 2 .

To obtain the refractive indexes when only two peaks are visible, we approximate the result considering that the delta shift $T_{1}=2 n_{0} d / c$ is equal to $T_{1}^{\prime}=$ $\left(3 n_{o}-n_{e}\right) d / c$, and the same with $T_{2}=2 n_{e} d / c$ and $T_{2}^{\prime}=\left(3 n_{e}-n_{0}\right) d / c$. Thus, the refractive index can be calculated from $T_{1}$ and $T_{2}$ as in the previous case.

In order to estimate the accuracy of the refractive index, the error propagation is applied to $n=T c / 2 m d$,

$$
E_{n}=\frac{T}{2 d m} E_{c}+\frac{c}{2 d m} E_{T}+\frac{c T}{2 d^{2} m} E_{d}
$$

where $E_{c}$ is the error of the light velocity approximation, $E_{d}$ is the measured error of the sample width, and $E_{T}$ is the absolute error caused by the temporal resolution achieved in the last DFT of the method, which depends on the terahertz broadband. When only two peaks are observable, the temporal resolution error increases for the reason that it is possible to measure $T_{1}$, instead of $T_{1}^{\prime}$ or otherwise, and the same with $T_{2}$ and $T_{2}^{\prime}$ (see Fig. 2). Therefore, the temporal resolution can be written as $E_{T}^{\prime}=E_{T}+\left|T_{1}^{\prime}-T_{1}\right|=E_{T}+\left|n_{o}-n_{e}\right| d / c=E_{T}+$ $\left|T_{1}-T_{2}\right| / 2$. In the numerical simulation of Fig. 2 , the refractive index error applying Eq. (4) is \pm 0.08 .

The proposed method can also be used to calculate the optical axis orientation of a birefringent material by

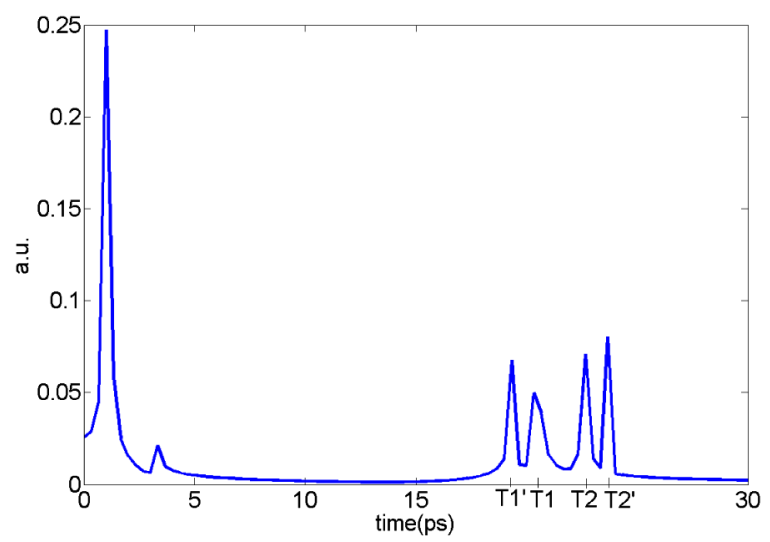

Fig. 2. Numerical simulation of the DFT applied to the frequency-domain signal of Eq. (2). 
making the amplitude ratio of the fourth and fifth terms of Eq. (3),

$$
\begin{aligned}
\frac{H_{4}}{H_{5}} & =\operatorname{tg}^{4} \theta \frac{\left(\frac{4 n_{e}}{\left(n_{e}+1\right)^{2}}\right)^{2} \frac{\left(n_{e}-1\right)^{2}}{\left(n_{e}+1\right)^{2}}}{\left(\frac{4 n_{o}}{\left(n_{o}+1\right)^{2}}\right)^{2} \frac{\left(n_{o}-1\right)^{2}}{\left(n_{o}+1\right)^{2}}} \\
\theta & \approx \operatorname{tg}^{-1}\left(\left(\frac{H_{4}}{H_{5}}\right)^{1 / 4}\right) .
\end{aligned}
$$

To know if the angle of the optical axis is clockwise or not, another measurement with a different angle of the optical axis has to be done. If $n_{o}$ is close to $n_{e}$, [Eq. ()] can be approximated by Eq. (ㅁ).

When only two peaks are discernible, the optical axis is calculated by making the ratio of the sum of the closer terms,

$$
\begin{gathered}
\frac{H_{4}+H_{2}}{H_{5}+H_{3}}=\operatorname{tg}^{2} \theta f\left(n_{o}, n_{e}, \theta\right), \\
\theta \approx \operatorname{tg}^{-1}\left(\frac{H_{4}+H_{2}}{H_{5}+H_{3}}\right)^{1 / 2},
\end{gathered}
$$

when $n_{o}$ is close to $n_{e}, f\left(n_{o}, n_{e}, \theta\right)$ is almost one, and Eq. (8) can be used to calculate the angle of the optical axis.

A numerical simulation of the absolute value of the DFT of Eq. (2) is presented in Fig. 3, assuming a bandwidth up to $1 \mathrm{THz}$ with a frequency resolution of $2.94 \mathrm{GHz}, n_{e}=3.07, n_{o}=3.39, d=1 \mathrm{~mm}$, and $\theta$ varying at $49.8^{\circ}, 54.6^{\circ}$, and $58.8^{\circ}$. It can be seen that only two peaks are observable due to the bandwidth of the signal in comparison to the case of Fig. 2.

The theoretical analysis assumes $\left|E_{i}(\omega)\right|^{2}$ is constant in frequency, but in a practical implementation the pulses will have a finite bandwidth that will broaden the pulses and vary the peaks' amplitudes. However, every peak changes its amplitude in the same proportion. Since the angle of the optical axis is derived from the amplitude ratio between peaks, the finite bandwidth is not an issue.

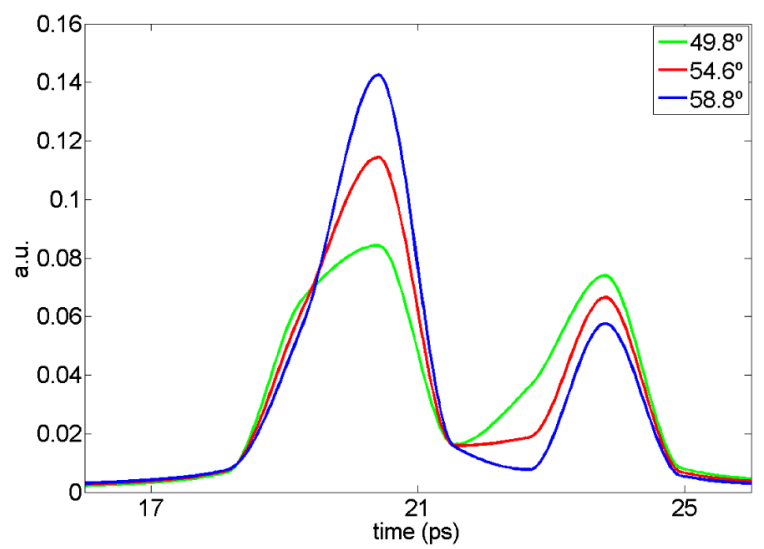

Fig. 3. Numerical simulation of the DFT applied to the frequency-domain signal [Eq. (2)].
To demonstrate the feasibility of the proposed method, the conventional terahertz TDS setup shown in Fig. 4 has been used. It is based on a mode-locked femtosecond laser, a pair of photoconductive antennas (PCA) based on LT-InGaAs/InAlAs, and a lock-in amplifier.

The sample used in the experiment was a sapphire plate of $1 \mathrm{~mm}$ width. The difference between the ordinary and extraordinary refractive index in sapphire is 0.32 [5] , producing a delay between the main pulses of $1 \mathrm{ps}$. A direct analysis in the time domain shows that the pulses corresponding to ordinary and extraordinary axes cannot be resolved [Fig. 5(a)].

The measurement was done with a time scan of $340 \mathrm{ps}$ and a sampling step of 200 fs. Figure 5(b) represents the result of making the squared modulus of the DFT of the time-domain signal [Fig. 5(a)] where the modulation caused by interference is observable. Finally, Fig. 5(c) shows the absolute modulus of the DFT of the signal presented in Fig. 5(b). Due to the limited bandwidth (i.e., the limited dynamic range) of the spectrometer setup, only two peaks can be observed. Nevertheless, the birefringence can be obtained $\left(T_{1}=20.2 \mathrm{ps}, \quad n_{1}=3.03\right.$, $\left.T_{2}=23.31 \mathrm{ps}, n_{2}=3.49\right)$. Near the origin, there are peaks caused by echoes generated in the system (appearing also without sample) and low-amplitude harmonic peaks around $45 \mathrm{ps}$, a consequence of the next series of echoes $(m=2)$. The results presented in Fig. 5(c) are in agreement with the published values of sapphire $[5,9]$.

The delay in Fig. $\underline{5(\mathrm{c})}$ is given by $\Delta t_{2}=T_{2}-T_{1}=$ $\left(2 n_{2}-2 n_{1}\right)(d / c)=2\left(\overline{n_{2}-n_{1}}\right)(d / c)$ which results in $2 \Delta t_{1}$; that is, applying the proposed data analysis, the time resolution is improved by a factor of 2 in comparison to the direct time-domain analysis.

Another set of experiments was carried out to assess the capability to determine the optical axis orientation. Figure 6 shows three measurements where the sample was rotated five grades each.

Using the peaks' amplitudes, the angles of the optical axis obtained applying Eq. (8) to the measurements of Fig. $\underline{6}$ agree quite well with the sample rotation angles, achieving a maximum difference of $1^{\circ}$ in the optical axis determination.

It is important to notice that for achieving an accurate result in the optical axis determination the emitter and the detector must be well aligned to apply Eqs. (6) and ( $\underline{8})$.

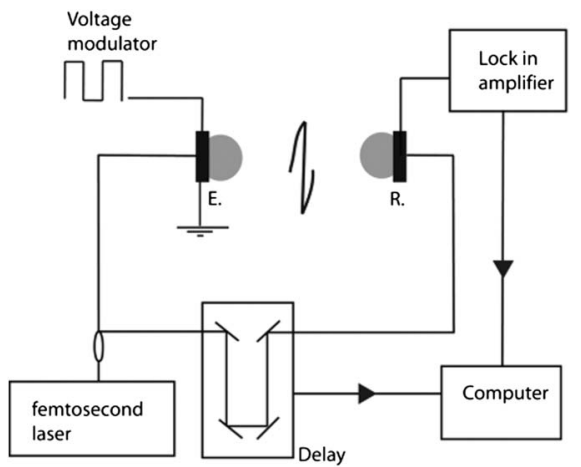

Fig. 4. Terahertz TDS spectrometer setup. 

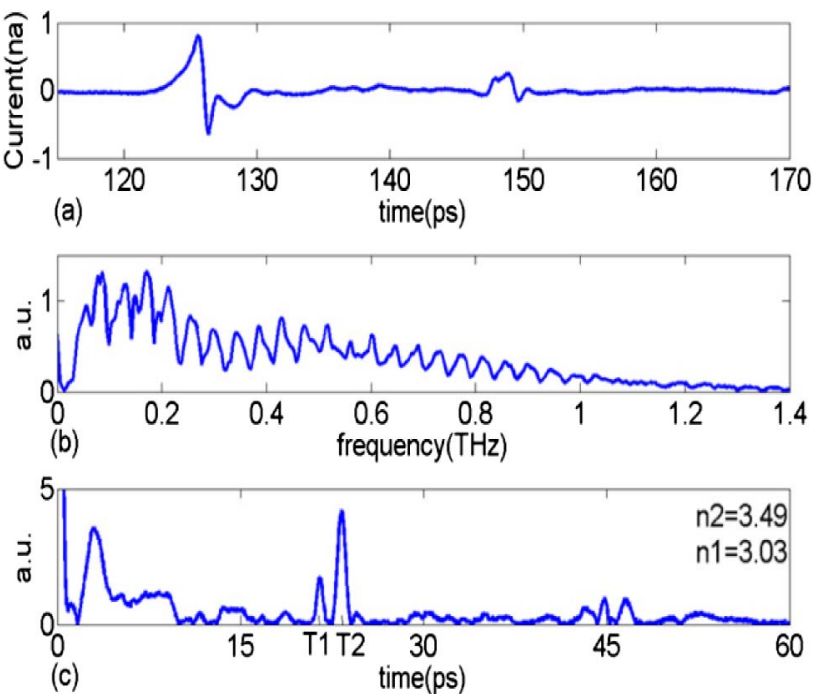

Fig. 5. (a) Temporal measurement of the terahertz spectrometer with a sapphire window of $1 \mathrm{~mm}$ and (b) modulus of the DFT applied to the signal in (a). (c) Squared modulus of the DFT applied to the signal in (b).

In Fig. 3 the numerical simulation was done with the same resolution, angles, material width, and refractive index values as the experiment. The theoretical simulation agrees quite well with the experimental results of Fig. $\underline{6}$. Differences are caused by the assumption of frequency independence of the refractive indices and $\left|E_{i}(w)\right|^{2}$ in Eq. (2).

In conclusion, a simple analysis for characterizing birefringence materials has been proposed. It allows the determination of the birefringence as well as the determination of the optical axis orientation even when diattenuation occurs. This method allows fast characterization in the terahertz band of transparent materials, such as plastics, paper, and wood without the need of a reference measurement and using a standard terahertz TDS setup.

The authors thank the Erasmus Mundus scholarship and the Consejo Nacional de Investigaciones Científicas y Técnicas (Conicet) that support the work of Federico Sanjuan at Universitat Politècnica de València and the Spanish Ministerio de Economia y Competitividad for partially supporting this work through project

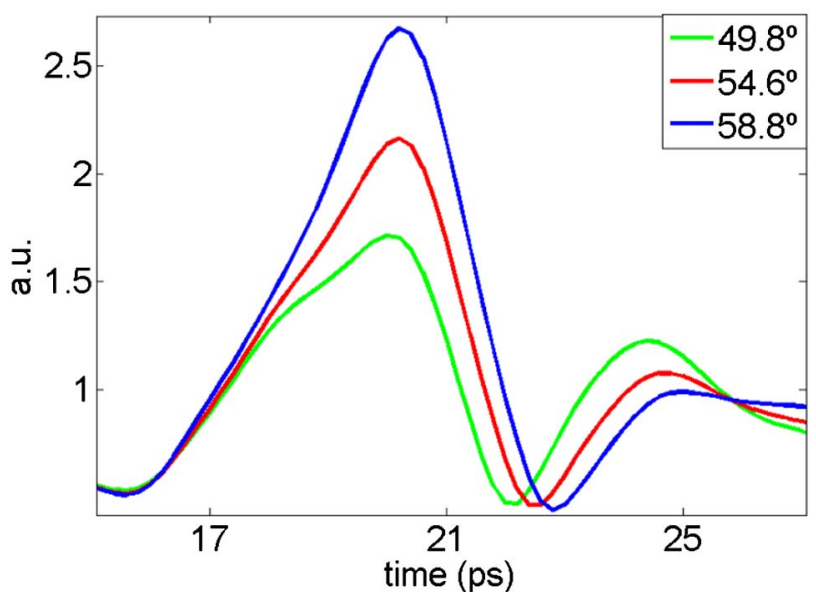

Fig. 6. Change in the DFT of the modulus of the terahertz spectrum when the sapphire sample mount is rotated in $5^{\circ}$ steps.

TEC2012-35797. Federico Sanjuan also thanks Dr. Jorge Tocho for his encouragement and support.

\section{References}

1. K. Wiesauer and C. Jördens, J. Infrared, Millimeter, Terahertz Waves 34, 663 (2013).

2. S. Ebara, Y. Hirota, M. Tani, and M. Hangyo, "Highly sensitive birefringence measurement in $\mathrm{THz}$ frequency region and its application to stress measurement," presented at the Joint 32nd International Conference on Infrared and Millimeter Waves and 15th International Conference on Terahertz Electronics (IRMMW-THz), Cardiff, Wales, September 2007.

3. C. Jördens, M. Scheller, S. Wietzke, D. Romeike, C. Jansen, T. Zentgraf, K. Wiesauer, V. Reisecker, and M. Koch, Compos. Sci. Technol. 70, 472(2010).

4. C. Jördens, M. Scheller, M. Wichmann, M. Mikulics, K. Wiesauer, and M. Koch, Appl. Opt. 48, 2037 (2009).

5. Y. Kim, M. Yi, B. Kim, and J. Ahn, Appl. Opt. 50, 2906 (2011).

6. M. Reid and R. Fedosejevs, Appl. Opt. 45, 2766 (2006).

7. S. Katletz, M. Pfleger, H. Uhringer, M. Mikulics, N. Vieweg, O. Peters, B. Scherger, M. Scheller, M. Koch, and K. Wiesauer, Opt. Express 20, 23025 (2012).

8. L. Duvillaret, F. Garet, and J. L. Coutaz, J. Opt. Soc. Am. B 17, 452 (2000).

9. D. Grischkowsky, S. Keiding, M. van Exter, and C. Fattinger, J. Opt. Soc. Am. B 7, 2006 (1990). 